\title{
Hypoglossal Nerve Mononeuropathy as the First Presenting Symptom of Progressing Multiple Myeloma
}

\author{
Neil B. Newman ${ }^{\text {a, c }}$, Vidya Puthenpura ${ }^{\text {b }}$,Stephanie Mischell ${ }^{\mathrm{a}}$, \\ Gabriela Ferreira $^{\text {a }}$
}

\begin{abstract}
Multiple myeloma (MM) rarely presents with a primary neurological dysfunction, and if it does it is usually due to a plasmacytoma. This is the first case to discuss hypoglossal nerve dysfunction as the first sign of $\mathrm{MM}$ progression secondary to severe pathophysiologic bone lysis. A PubMed-based literature search was completed on April 17, 2016 for the terms "multiple myeloma" and "hypoglossal nerve neuropathy". A 73-year-old woman with known MM who received little treatment for several years, presented secondary to dysarthria and at first was thought to have hyperviscosity syndrome. On further examination, it was found she had light chain disease and her symptoms were secondary to severe disease progression. Imaging revealed multiple lytic lesions in the skull on skeletal survey and brain MRI revealed boney lysis near the occipital condyle and clivus likely interfering with the coursing of the hypoglossal nerve. Advanced progressing MM can cause severe boney destruction which can interfere with cranial nerve canals and cause neuropathy as a presenting symptom.
\end{abstract}

Keywords: Multiple myeloma; Hypoglossal nerve palsy

\section{Introduction}

Multiple myeloma (MM) is a heterogeneous clonal plasma cell dyscrasia [1] that comprises merely $10 \%$ of hematologic malignancies [2]. The classic complications of myeloma are well studied with renal failure, anemia, hypercalcemia, and bone pains as the most well recognized [3]. Neurological findings are less common and can present secondary to peripheral neuropathy or as an epidural plasmacytoma causing compres-

Manuscript accepted for publication January 10, 2017

a Department of Medicine, Rutgers Robert Wood Johnson Medical School, New Brunswick, NJ 07082, USA

bDepartment of Pediatrics, Rutgers Robert Wood Johnson Medical School, New Brunswick, NJ 07082, USA

${ }^{\mathrm{c} C}$ Corresponding Author: Neil Newman, Department of Medicine, Rutgers Robert Wood Johnson Medical School, New Brunswick, NJ 07082, USA. Email: nbn426@gmail.com

doi: https://doi.org/10.14740/wjon1000w sion of peripheral nerves [4]. Cranial nerve impingement of the fifth [5] or sixth nerve [5] has been described in MM, most often secondary to a soft tissue mass causing nerve compression. Compression of the hypoglossal nerve has been reported [6], albeit rarely, and when reported it is usually secondary to a plasmacytoma [7]. Besides a case of jaw numbness [8] secondary to myeloma affecting the jaw bones, there are no cases discussing cranial nerve neuropathy secondary to severe bone remodeling caused by advanced MM.

In this report, we describe a case of a woman with known MM whose only presenting symptom of disease progression was a deviated tongue. While at first this was thought to be a stroke secondary to hyperviscosity syndrome, it ended up due to severe cranial deformities at the ocular condyle near the coursing of the hypoglossal nerve affecting its function.

A PubMed-based literature search was completed on April 17, 2016 for the terms "multiple myeloma" and "hypoglossal nerve neuropathy".

\section{Case Report}

A 73-year-old female with a history of MM presented to the emergency department with progressive slurred speech due to a rightward deviated tongue for 4 days. These symptoms were not associated with vision loss or weakness. Physical exam demonstrated overt right-sided tongue deviation, with no other focal neurologic deficits. The patient endorsed a history of MM diagnosis in the past but underwent only a few chemotherapy treatments due to her religious beliefs as a Jehovah's Witness and was generally not receiving regular medical care. Given the patient's history of MM, a code stroke was initiated for suspected hyperviscosity syndrome as the cause of the stroke. She received a computed tomography (CT) of her head, which revealed marrow abnormalities specifically in the calvarium consistent with advanced MM, but no clear areas of ischemic or hemorrhagic strokes. The clinicians decided to further pursue a magnetic resonance angiogram (MRA) of her brain, which revealed a patent circle of Willis with no occlusions. After obtaining more collateral information, it was revealed the patient was diagnosed with MM in 2008, and was found at that time to have $20 \%$ plasma cells in her marrow. She received several cycles of chemotherapy at that time but chose not to continue therapy. In 2010, she received radiation 


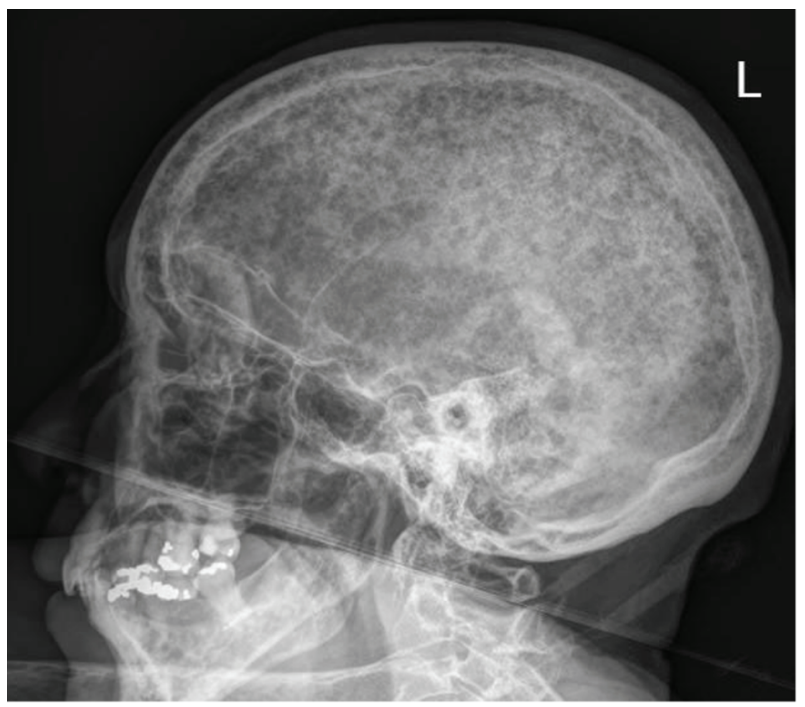

Figure 1. Skeletal survey of the skull demonstrating extensive punched out lesions consistent with advanced disease.

for two plasmacytomas that formed in the epidural space but had limited follow-up or further treatment thereafter.

On further evaluation, the patient was found to have hypercalcemia with a level of $12.1 \mathrm{mg} / \mathrm{dL}$ (14 taking into account correction for hypoalbuminemia), anemia with a hemoglobin level of $6.5 \mathrm{mg} / \mathrm{dL}$, and kidney failure with a creatinine level of 4.7. She also had a $\beta 2$ microglobulin level greater than 10 $\mathrm{mg} / \mathrm{L}$ consistent with stage III MM per Durie-Salmon criteria. Hemodialysis and chemotherapy were offered to the patient; however, the patient refused. Serum viscosity levels were found to be within normal limits. Serum immunofixation demonstrated that the free lambda clone was present, with no corresponding $\operatorname{IgG}, \operatorname{IgA}$ or $\operatorname{IgM}$ heavy chains, clarifying that she had light chain myeloma.

The skeletal survey seen in Figure 1 demonstrated the significant progression of her disease, especially near the skull base. Magnetic resonance imaging (MRI) of her brain, seen in Figure 2, revealed that the patient had myelomatous lesions at the right occipital condyle and clivus consistent with MM disease advancement. The cause of her dysarthria was concluded to be hypoglossal nerve mononeuropathy, due to impingement as the nerve courses through the hypoglossal canal, located at the base of the right occipital condyle. Although the patient did understand that all of her conditions stemmed from poorly controlled disease, she did not desire to pursue further chemotherapy or receive blood transfusions at the time.

\section{Discussion}

Untreated MM can have several serious side effects as this case reveals. MM causes severe osteolytic destruction via cytokines, specifically IL-1, which then activates RANK-L on osteoclasts [9]. When the disease is markedly uncontrolled, as was revealed in this case, there is marked bone remodeling and lysis throughout the body. Impingement of the hypoglos-

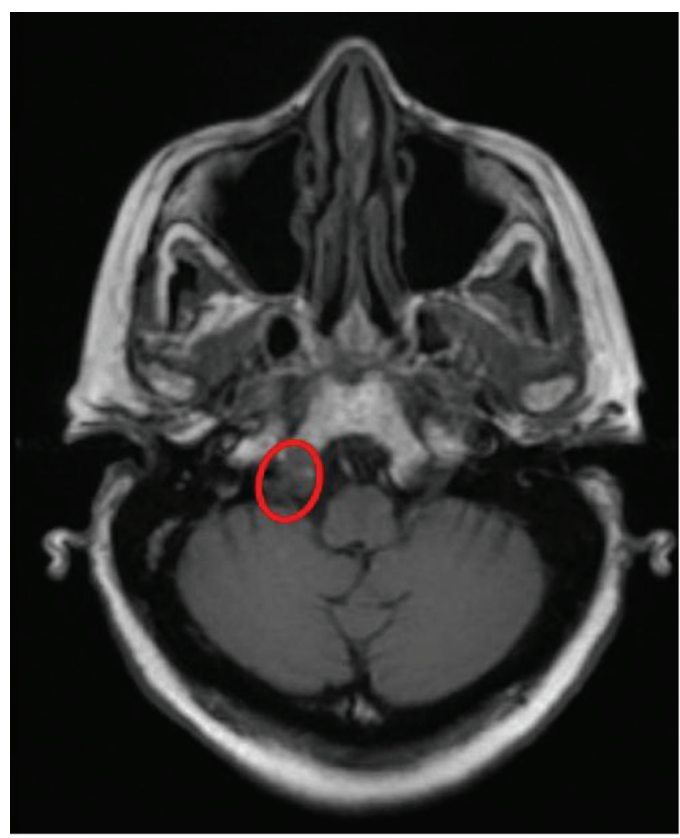

Figure 2. Eroded occipital condyle affecting the hypoglossal nerve.

sal nerve causing mononeuropathy at the occipital condyle has been reported in association with condyle fracture [10]. Furthermore, a review article focusing on MRI of the course of the hypoglossal nerve demonstrates how any interruption of the nerve course at the clivus or condyle due to pathologies such as bone destruction or tumor invasion leads to the mononeuropathy [11]. Similarly, this patient had severe osteopenia and marrow involvement at both the right clivus and right occipital condyle. This involvement directly explains the right-sided (lower motor neuron) pathology visualized on exam.

This case has clinical value for several reasons. The literature has reports of cranial nerve neuropathies caused by MM secondary to plasmacytoma (solid soft tissue mass of myeloma cells) compression of CN III [12], myelomatous meningitis [4], and hyperviscosity syndrome [13]. This is the first case that the authors are aware of in the literature where a cranial nerve abnormality has resulted secondary to pathophysiological sequelae of the disease. Furthermore, this case is of clinical value because the hypoglossal nerve impingement was the first presenting symptom of marked disease advancement. Presumably her other clinical symptoms, including renal, hematologic, and/or electrolyte imbalances would have manifested in some other way prior to this presentation. It also speaks towards the importance of mirroring to patients the manifestations of untreated disease. While this patient was not open to certain therapies such as blood transfusions, she did not seem to grasp that her cancer could markedly affect her bone formation, which ultimately led to her chief complaint of slurred speech and right tongue deviation.

\section{Conclusions}

Uncontrolled MM can affect the skull and coursings of cranial 
nerves. Awareness of these sequelae of unmanaged MM can help in more appropriate diagnosis of a patient.

\section{References}

1. Palumbo A, Anderson K. Multiple myeloma. N Engl J Med. 2011;364(11):1046-1060.

2. Kariyawasan CC, Hughes DA, Jayatillake MM, Mehta AB. Multiple myeloma: causes and consequences of delay in diagnosis. QJM. 2007;100(10):635-640.

3. Kyle RA, Gertz MA, Witzig TE, Lust JA, Lacy MQ, Dispenzieri A, Fonseca R, et al. Review of 1027 patients with newly diagnosed multiple myeloma. Mayo Clin Proc. 2003;78(1):21-33.

4. van Ginkel S, Snijders TJ, van de Donk NW, Klijn CJ, Broekman ML. Progressive neurological deficits in multiple myeloma: meningeal myelomatosis without MRI abnormalities. J Neurol. 2012;259(6):1231-1233.

5. Thiruvengadam SS, Prayson RA. Multiple myeloma presenting with unilateral abducens and trigeminal nerve palsies. J Clin Neurosci. 2016;26:143-144.

6. Tucker D, Musuka C. Isolated 12th cranial nerve palsy as a complication of multiple myeloma. Br J Haematol. 2004;124(1):1.

7. Sin WY, Grant I. Intracranial plasmacytoma with hypoglossal nerve palsy in multiple myeloma. Postgrad Med J. 2015;91(1073):174-175.

8. Elias HG, Scott J, Metheny L, Quereshy FA. Multiple myeloma presenting as mandibular ill-defined radiolucent lesion with numb chin syndrome: a case report. J Oral Maxillofac Surg. 2009;67(9):1991-1996.

9. Abe M. [Myeloma bone disease]. Clin Calcium. 2014;24(8):1159-1168.

10. Orbay T, Aykol S, Seckin Z, Ergun R. Late hypoglossal nerve palsy following fracture of the occipital condyle. Surg Neurol. 1989;31(5):402-404.

11. Thompson EO, Smoker WR. Hypoglossal nerve palsy: a segmental approach. Radiographics. 1994;14(5):939-958.

12. Kalwani N, Remenschneider AK, Faquin W, Ferry J, Holbrook EH. Plasmacytoma of the Clivus Presenting as Bilateral Sixth Nerve Palsy. J Neurol Surg Rep. 2015;76(1):e156-159.

13. Panda BB, Parija S, Mallick J, Pujahari S. Oculomotor Nerve Palsy as a Rare Presentation and First Sign of Multiple Myeloma. J Clin Diagn Res. 2016;10(5):ND01-03. 